\title{
Moyamoya Syndrome Associated With Gamma Knife Surgery for Cerebral Arteriovenous Malformation
}

\author{
-Case Report-
}

\author{
Yoichi UOzUmi, ${ }^{1,2}$ Masaki SUMITOMO, ${ }^{2}$ Mikio MARUWAKA, ${ }^{2}$ Yoshio ARAKI, ${ }^{2}$ \\ Takashi IZUMI, ${ }^{2}$ Shigeru MIYACHI, ${ }^{2}$ Takenori KATO, ${ }^{3}$ Toshinori HASEgAWA, ${ }^{3}$ \\ Yoshihisa KIDA, ${ }^{3}$ Sho OKAMOTO, ${ }^{2}$ and Toshihiko WAKABAYASHI ${ }^{2}$
}

\begin{abstract}
${ }^{1}$ Department of Neurosurgery, Kamiiida Daiichi General Hospital, Nagoya, Aichi; ${ }^{2}$ Department of Neurosurgery, Nagoya University, Graduate School of Medicine, Nagoya, Aichi; ${ }^{3}$ Department of Neurosurgery, Gamma Knife Center, Komaki City Hospital, Komaki, Aichi
\end{abstract}

\begin{abstract}
A 30-year-old female developed moyamoya syndrome after gamma knife surgery (GKS) for cerebral arteriovenous malformation (AVM), and was treated with bypass surgery. She suffered from flittering scotoma, right transient hemianopsia, and headache for 1 year. Cerebral angiography revealed a Spetzler-Martin grade III AVM located in the left occipital lobe. After staged embolization, GKS was performed with a minimum dose of $20 \mathrm{~Gy}$ to the periphery of the nidus at the $50 \%$ isodose level of the maximum target dose. Gradual nidus regression was achieved, and the clinical symptoms disappeared completely. However, at 30 months after GKS, the patient suffered transient ischemic attack. Cerebral angiography showed left middle cerebral artery occlusion with moyamoya vessels. The patient underwent direct and indirect bypass surgery. After surgery, the patient was free from ischemic symptoms. Chronic inflammation and long-term changes in expression of cytokines and growth factors after GKS may have triggered this case.
\end{abstract}

Key words: moyamoya syndrome, gamma knife surgery, arteriovenous malformation, revascularization

\section{Introduction}

Gamma knife surgery (GKS) is an important therapeutic modality for cerebral arteriovenous malformation (AVM). ${ }^{7,9)}$ Progressive intracranial artery occlusion due to radiation-induced vasculopathy is a serious side effect of conventional radiation therapy for intracranial disease, ${ }^{2,8,12)}$ but moyamoya syndrome is very rare after GKS. ${ }^{17)}$ Here we report a case of moyamoya syndrome associated with GKS performed for AVM, which was treated by bypass surgery.

\section{Case Presentation}

A 30-year-old female suffered from flittering scotoma, right transient hemianopsia, and headache for 1 year. She was admitted to our institution. Cerebral angiography revealed a Spetzler-Martin grade III AVM located in the left occipital lobe and splenium of the corpus callosum (Fig. 1A, B). Staged embolization of the left posterior cerebral artery and anterior cerebral artery was performed. The nidus was reduced by $30 \%$ (Fig. 1C, D). Angiography before

Received October 6, 2011; Accepted February 10, 2012 and after the embolization detected no focal stenosis of the intracranial vessels. After embolization, GKS was performed with a minimum dose of $20 \mathrm{~Gy}$ to the periphery of the AVM nidus at the $50 \%$ isodose level of the maximum target dose (Fig. 2A). The nidus volume was estimated as $16.6 \mathrm{ml}$. The right internal carotid artery (ICA) terminal, right middle cerebral artery (MCA) M1 segment, left ICA terminal, and left MCA M1 segment received 1.4, 1.0, 2.1, and $1.9 \mathrm{~Gy}$, respectively. Gradual nidus regression was observed on serial follow-up brain magnetic resonance (MR) imaging and cerebral angiography. Her clinical symptoms disappeared completely. However, at 30 months after GKS, the patient began to suffer from transient right hemiparesis.

Technetium-99m-ethylcysteinate dimer ( $\left.{ }^{99 \mathrm{~m}} \mathrm{Tc}-\mathrm{ECD}\right)$ single photon emission computed tomography (SPECT) at rest demonstrated low cerebral blood flow (CBF) in the left cerebral hemisphere (Fig. 2C). Cerebral angiography showed occlusion of the left MCA M1 segment with moyamoya-like vessels. No anastomosis extended from the external carotid arteries to the intracranial arteries (Fig. $2 \mathrm{~B}, \mathrm{D})$. The symptoms were considered to be transient ischemic attack. Direct and indirect bypass surgery consisting of superficial temporal artery (STA)-MCA anasto- 

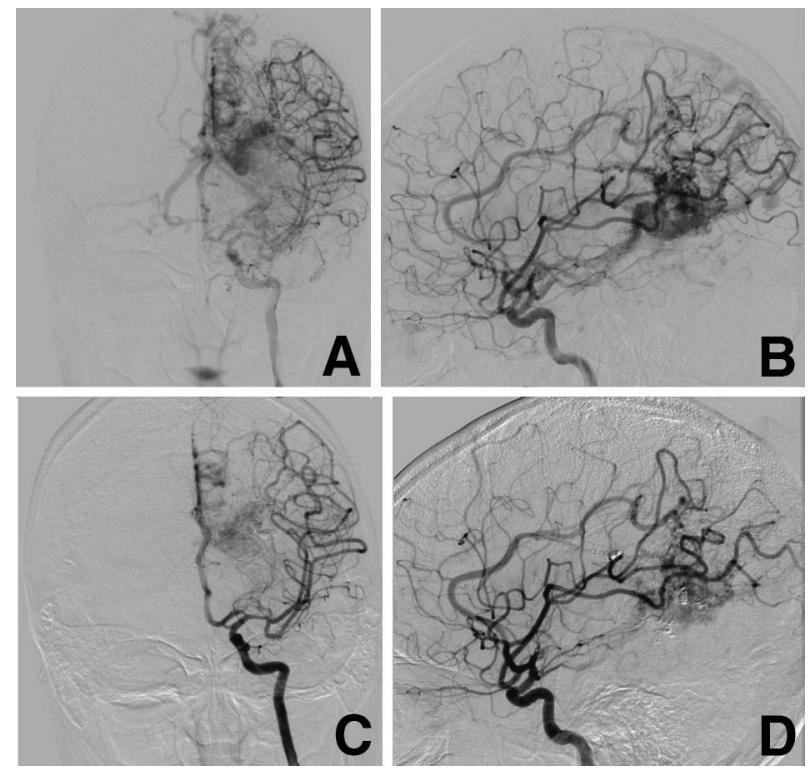

Fig. 1 A, B: Left carotid angiograms, anteroposterior (A) and lateral views (B), revealing a Spetzler-Martin grade III arteriovenous malformation located in the left occipital lobe and splenium of the corpus callosum fed by the left anterior, middle, and posterior cerebral arteries. C, D: Left carotid angiograms, anteroposterior (C) and lateral views (D), after the second transarterial embolization showing some nidus reduction.
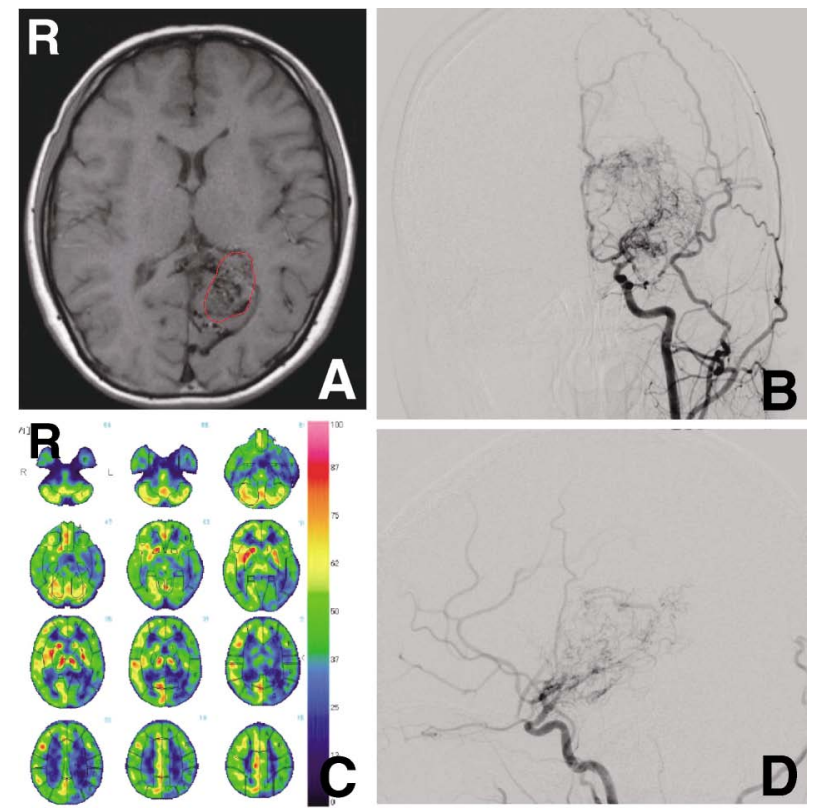

Fig. 2 A: $T_{1}$-weighted magnetic resonance image indicating the scheduled dose plan. B, D: Left carotid angiograms, anteroposterior (B) and lateral views (D), at 21 months after gamma knife surgery showing proximal middle cerebral artery occlusion with moyamoya-like vessels forming the internal carotid artery bifurcation. C: Technetium-99m-ethylcysteinate dimer single photon emission computed tomography scans at rest demonstrating low cerebral blood flow in the left cerebral hemisphere.

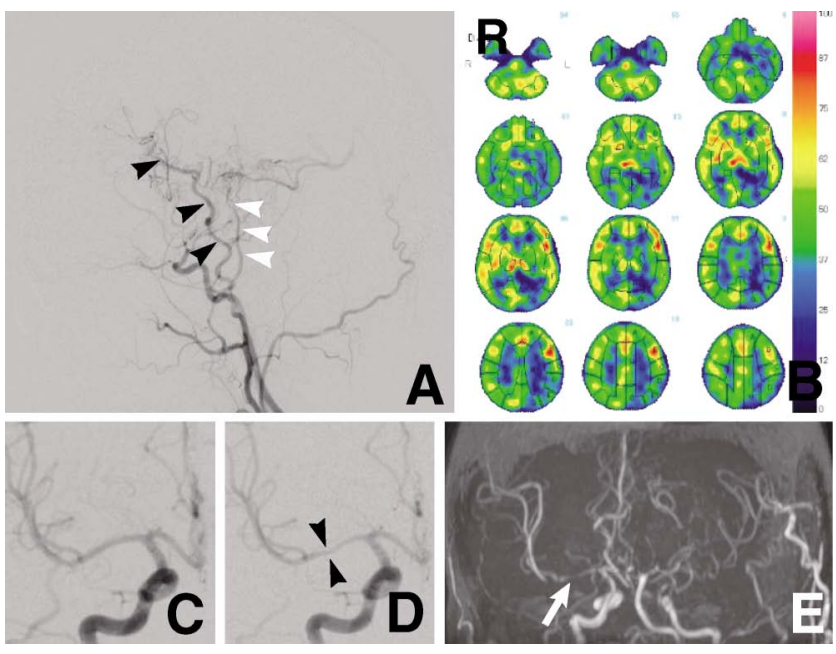

Fig. 3 A: Left carotid angiogram at 12 months after the surgery confirming adequate bypass flow in the frontal and temporal branches of the superficial temporal artery to the frontal and temporal lobes (black and white arrowheads), respectively. B: Technetium-99m-ethylcysteinate dimer single photon emission computed tomography scans at rest after surgery demonstrating improved cerebral blood flow in the left cerebral hemisphere. C, D: Serial left carotid angiograms at 31 (C) and 43 months (D) after gamma knife surgery (GKS) revealing progression of the right M1 stenosis (arrowheads). E: Magnetic resonance angiogram at 46 months after GKS showing right severe M1 stenosis (arrow).

mosis and encephalo-myo-synangiosis were performed on the left.

After surgery, the patient became free from ischemic symptoms. Adequate bypass flow was confirmed by follow-up angiography and ${ }^{99 m}$ Tc-ECD SPECT at rest (Fig. $3 \mathrm{~A}, \mathrm{~B})$. Serial angiography revealed progression of the right M1 stenosis (Fig. 3C, D). At 47 months after GKS, MR angiography showed severe right M1 stenosis (Fig. $3 \mathrm{E})$. The patient remained neurologically stable and was free from clinical symptoms. Further observation is needed.

\section{Discussion}

This is an extremely rare case of moyamoya syndrome associated with GKS for AVM treated with bypass surgery. AVM associated with moyamoya disease is rare. Most cases had both neurovascular anomalies at the first presentation. ${ }^{1,3,6,10,11,13,16)}$ High CBF related to the AVM may cause hemodynamic stress and affect the endothelium of the circle of Willis. ${ }^{10,11)}$ Some cases of moyamoya disease show de novo development of AVM in the followup periods. ${ }^{4,15)}$ In these cases, angiogenesis factors such as vascular endothelial growth factor (VEGF) might be activated by developing pial collateral formation, and subsequently induced AVM formation. ${ }^{16)}$ Only two cases have shown de novo development of moyamoya vessels within the follow-up period of several years after treatment for AVMs (including our case). ${ }^{16)}$ 
Our present patient and the previous patient in Taiwan developed bilateral moyamoya vessels after GKS. The high directional characteristic of GKS helps to avoid irradiation to the area surrounding the lesion. Our patient received 1.0-2.1 Gy at each ICA terminal and MCA M1 segment. Most cases of radiation-induced moyamoya syndrome received 22-120 Gy (mean $55.2 \mathrm{~Gy}$ ). ${ }^{2,8}$ ) These patients were treated with conventional radiation therapy for parasellar brain tumor. The irradiation field included the ICA terminal and the MCA M1 segment. Radiation-induced moyamoya syndrome is likely to occur within the irradiation field after high dose radiation therapy. Therefore, direct radiation injury may not be the main cause of moyamoya syndrome after GKS for AVM. Recently, radiation therapy was suggested to induce chronic oxidative stress and inflammation, ${ }^{18)}$ and irradiated tissue may undergo long-term changes in the expression of cytokines and growth factors. ${ }^{5}$ The guidelines of research on intractable diseases of the Ministry of Health Labour and Welfare, Japan, suggest that inflammation leads to moyamoya syndrome. ${ }^{14)}$ Therefore, chronic inflammation due to GKS may have caused, in part, the formation of moyamoya vessels in this case. The long-term follow up of this case and accumulation of similar cases are required to resolve the mechanism of pathogenesis.

The treatment strategy of this case is controversial. Bypass surgery was performed because the patient suffered from transient ischemic attack after GKS and decreased CBF. However, this procedure carried some risk of AVM progression and rupture due to the nidus flow increase from the bypass flow. AVM progression has been reported after STA-MCA anastomosis for moyamoya disease. ${ }^{6)}$ Our case must be followed up in the long term to detect this rare complication.

\section{References}

1) Ahn SH, Choo IS, Kim JH, Kim HW: Arteriovenous malformation with an occlusive feeding artery coexisting with unilateral moyamoya disease. J Clin Neurol 6: 216-220, 2010

2) Desai SS, Paulino AC, Mai WY, Teh BS: Radiation-induced moyamoya syndrome. Int J Radiat Oncol Biol Phys 65: 1222-1227, 2006

3) Enam SA, Malik GM: Association of cerebral arteriovenous malformations and spontaneous occlusion of major feeding arteries: clinical and therapeutic implications. Neurosurgery 45: 1105-1111, 1999

4) Fuse T, Takagi T, Fukushima T, Hashimoto N, Yamada K: Arteriovenous malformation associated with moyamoya disease. Childs Nerv Syst 12: 404-408, 1996

5) Gallet P, Phulpin B, Merlin JL, Leroux A, Bravettti P, Mecellem H, Tran N, Dolivet G: Long-term alterations of cytokines and growth factors expression in irradiated tis- sues and relation with histological severity scoring. PLoS One 6: e29399, 2011

6) Halatsch ME, Rustenbeck HH, Jansen J: Progression of arteriovenous malformation in moyamoya syndrome. Acta Neurochir (Wien) 139: 82-85, 1997

7) Karlsson B, Lindquist C, Steiner L: Prediction of obliteration after gamma knife surgery for cerebral arteriovenous malformations. Neurosurgery 40: 425-430, 1997

8) Kestle JR, Hoffman HJ, Mock AR: Moyamoya phenomenon after radiation for optic glioma. J Neurosurg 79: 32-35, 1993

9) Maruyama K, Kawahara N, Shin M, Tago M, Kishimoto J, Kurita H, Kawamoto S, Morita A, Kirino T: The risk of hemorrhage after radiosurgery for cerebral arteriovenous malformations. N Engl J Med 352: 146-153, 2005

10) Mawad ME, Hilal SK, Michelsen WJ, Stein B, Ganti SR: Occlusive vascular disease associated with cerebral arteriovenous malformations. Radiology 153: 401-408, 1984

11) Montanera W, Marotta TR, terBrugge KG, Lasjaunias $P$, Willinsky R, Wallace MC: Cerebral arteriovenous malformations associated with moyamoya phenomenon. AJNR Am J Neuroradiol 11: 1153-1156, 1990

12) Morris B, Partap S, Yeom K, Gibbs IC, Fisher PG, King AA: Cerebrovascular disease in childhood cancer survivors: A Children's Oncology Group Report. Neurology 73: 1906-1913, 2009

13) Okada T, Kida Y, Kinomoto T, Sakurai T, Kobayashi T: Arteriovenous malformation associated with moyamoya disease-case report. Neurol Med Chir (Tokyo) 30: 945-948, 1990

14) Research on intractable diseases of the Ministry of Health, Labour and Welfare, Japan: [Recommendations for the management of moyamoya disease. A statement from Research Committee on Spontaneous Occlusion of the Circle of Willis (Moyamoya Disease)]. No Sotchu No Geka 37: 321-337, 2009 (Japanese)

15) Schmit BP, Burrows PE, Kuban K, Goumnerova L, Scott RM: Acquired cerebral arteriovenous malformation in a child with moyamoya disease. Case report. J Neurosurg 84: 677-680, 1996

16) Seol HJ, Kim DG, Oh CW, Han DH: Radiosurgical treatment of a cerebral arteriovenous malformation in a patient with moyamoya disease: case report. Neurosurgery 51: 478-481, 2002

17) Wu TC, Guo WY, Wu HM, Chang FC, Shiau CY, Chung WY: The rare association of moyamoya disease and cerebral arteriovenous malformations: a case report. Korean J Radiol 9 Suppl: S65-S67, 2008

18) Zhao W, Robbins ME: Inflammation and chronic oxidative stress in radiation-induced late normal tissue injury: therapeutic implications. Curr Med Chem 16: 130-143, 2009

Address reprint requests to: Sho Okamoto, MD, PhD, Department of Neurosurgery, Nagoya University, Graduate School of Medicine, 65 Tsurumai-cho, Showa-ku, Nagoya, Aichi 446-8550, Japan.

e-mail: soknouge@med.nagoya-u.ac.jp 